\section{BMJ Paediatrics Open}

\title{
Early preterm nutrition and the urinary metabolome in young adult life: follow- up of a randomised controlled trial
}

James R C Parkinson, ${ }^{1}$ Anisha D Wijeyesekera, ${ }^{2}$ Matthew J Hyde, ${ }^{1}$ Atul Singhal, ${ }^{3}$ Alan Lucas, ${ }^{3}$ Elaine Holmes, ${ }^{2}$ Neena Modi ${ }^{1}$

To cite: Parkinson JRC, Wijeyesekera AD, Hyde MJ, et al. Early preterm nutrition and the urinary metabolome in young adult life: follow-up of a randomised controlled trial. BMJ Paediatrics Open 2017;1:e000192. doi:10.1136/ bmjpo-2017-000192

- Additional material is published online only. To view, please visit the journal online (http://dx.doi.org/10.1136/ bmjpo-2017-000192).

JRCP and ADW contributed equally.

Received 8 August 2017 Revised 5 October 2017 Accepted 6 October 2017

\section{(a) CrossMark}

${ }^{1}$ Section of Neonatal Medicine, Imperial College London, London, UK

${ }^{2}$ Division of Computational and Systems Medicine, Department of Surgery and Cancer, Imperial College London, London, UK ${ }^{3}$ Department of Nutrition, Institute of Child Health, London, UK

Correspondence to

Professor Neena Modi; n.modi@ imperial.ac.uk

\section{ABSTRACT}

Objective We aimed to test the hypothesis that early diet programmes the metabolic profile of young adults born preterm.

Design We analysed banked urine samples obtained at a 20-year follow-up visit from adults that had participated as neonates in controlled trials involving randomisation within 48 hours of birth to feeds of preterm formula (PTF), banked breast milk (BBM) or term formula (TF) for 1 month postnatally.

Main outcome measures We performed proton nuclear magnetic resonance spectroscopy, analysing spectra by dietary group and sex. Orthogonal projections to latent structure discriminant analyses was used to model class differences and identify metabolites contributing to the differences between groups. Additionally, spectra were correlated with birth weight, gestational age and weight $z$ score at 2 weeks of age.

Results of the original number of 926 trial participants, urine samples were available from $197(21 \%)$ healthy young adults ( $42 \%$ men) born preterm (mean $30.7 \pm 2.8$ weeks) and randomised to BBM ( $n=55 ; 28$ men), TF ( $n=48$; 14 men) and PTF ( $n=93 ; 40$ men). We found no significant differences in urinary spectra between dietary groups including when stratified by sex. Correlation analysis revealed a weak association between metabolic profile and gestational age that was lost on controlling for ethanol excretion.

Conclusions We found no evidence that dietary exposures in the neonatal period influence the metabolic phenotype in young adult life.

\section{INTRODUCTION}

There is a growing body of evidence indicating that preterm birth is a risk factor for adverse long-term health. ${ }^{12}$ What is not known is the extent to which these are consequent on prematurity, or to the treatments received during neonatal care. It has been suggested that critical periods of prenatal and postnatal development exist, during which time nutrition appears to influence long-term disease risk. ${ }^{3} 4$ Cross-sectional studies show that preterm and low birthweight infants are at risk of later type 2 diabetes, hypertension, heart disease and visceral adiposity, and that

\section{What is already known on this topic?}

Growing evidence indicates preterm birth is a risk factor for adverse long-term health.

- Cross-sectional studies show that preterm and low birthweight infants are at risk of later type 2 diabetes, hypertension, heart disease and visceral adiposity.

- While beneficial to neurocognitive and motor development, a higher nutrient postnatal diet in preterm infants leads to an increase in developing metabolic syndrome-associated features.

- We have previously reported differences in the urinary metabolome of healthy young adult men born either very preterm or at term.

\section{What this study adds?}

We found no evidence of an effect of neonatal diet, allocated by randomisation, on the urinary metabolome of ex-preterm adults.

- Correlation analysis between metabolic spectra and birth weight, gestational age and 2-week weight z score also found no reliable associations.

- Our study indicates that recommendations for future work include standardisation of urine collection and consideration of power to detect prespecified and sex-specific differences.

rapid postnatal growth may be a contributory factor. $^{5-8}$

In the early 1980s, Lucas established two interventional trials in which preterm infants $(<37$ weeks gestation) were randomised to receive either higher (preterm formula (PTF) ) or lower energy density feeds (banked breast milk (BBM); term formula (TF)) for an average of 1 month. ${ }^{9}{ }^{10}$ Longitudinal follow-up of this cohort revealed significant differences in a number of key metabolic outcomes. Higher nutrient intake (PTF) resulted in greater growth velocity during the month-long intervention ${ }^{11}$ and improved motor and mental development ${ }^{9} 1213$ when 
compared with lower nutrient groups (TF, BBM). In childhood and adolescence, PTF was associated with increased blood pressure compared with both $\mathrm{TF}^{6}$ and $\mathrm{BBM}^{14}$ groups. Furthermore, at the age of 13-16 years participants randomised to PTF showed evidence of insulin ${ }^{15}$ and leptin resistance. ${ }^{16}$ These data indicate that while apparently beneficial to neurocognitive and motor development, a higher nutrient postnatal diet resulted in an increase in features of metabolic syndrome in later life.

In this study, a post hoc exploratory analysis, we aimed to apply untargeted metabolic profiling using proton nuclear magnetic resonance ( $\left.{ }^{1} \mathrm{H}-\mathrm{NMR}\right)$ spectroscopy to investigate the relationship between neonatal nutritional exposures and the urinary metabolic profile in young adult life. Untargeted metabolic profiling enables highthroughput detection of low molecular weight molecules present in biological samples at any given moment in time. ${ }^{17}$ Metabolites captured in these samples include intermediate and end products of endogenous metabolic pathways, and exogenous signals arising from diet, drugs and other lifestyle and environmental stimuli. Thus, this systems approach facilitates better understanding of genetic, lifestyle, environment and other pathophysiological stimuli on health. We used these urinary metabolic profiles to assess whether early life diet shapes health in young adulthood and examine the relationship with gestational age, birth weight and growth velocity as these are potential effect modifiers.

\section{METHODS}

Study design and sample collection

The initial study involved recruitment of infants born at $<37$ weeks completed gestation and with a birth weight $<1850 \mathrm{~g}$, and was conducted in five neonatal units in the UK between 1982 and $1985 .{ }^{11}$ The study design is outlined in figure 1 . The trial protocol and subsequent follow-up evaluations have been published extensively. ${ }^{29-1113-1618}$ No registration is available as the original trial was conducted in the early 1980s, at a time when registration was not common. A balanced randomisation sequence was prepared for each centre, within strata defined by birth weight: $<1200 \mathrm{~g}$ and $1200-1849 \mathrm{~g}$. Treatment assignments were held in sealed numbered envelopes. ${ }^{11}$

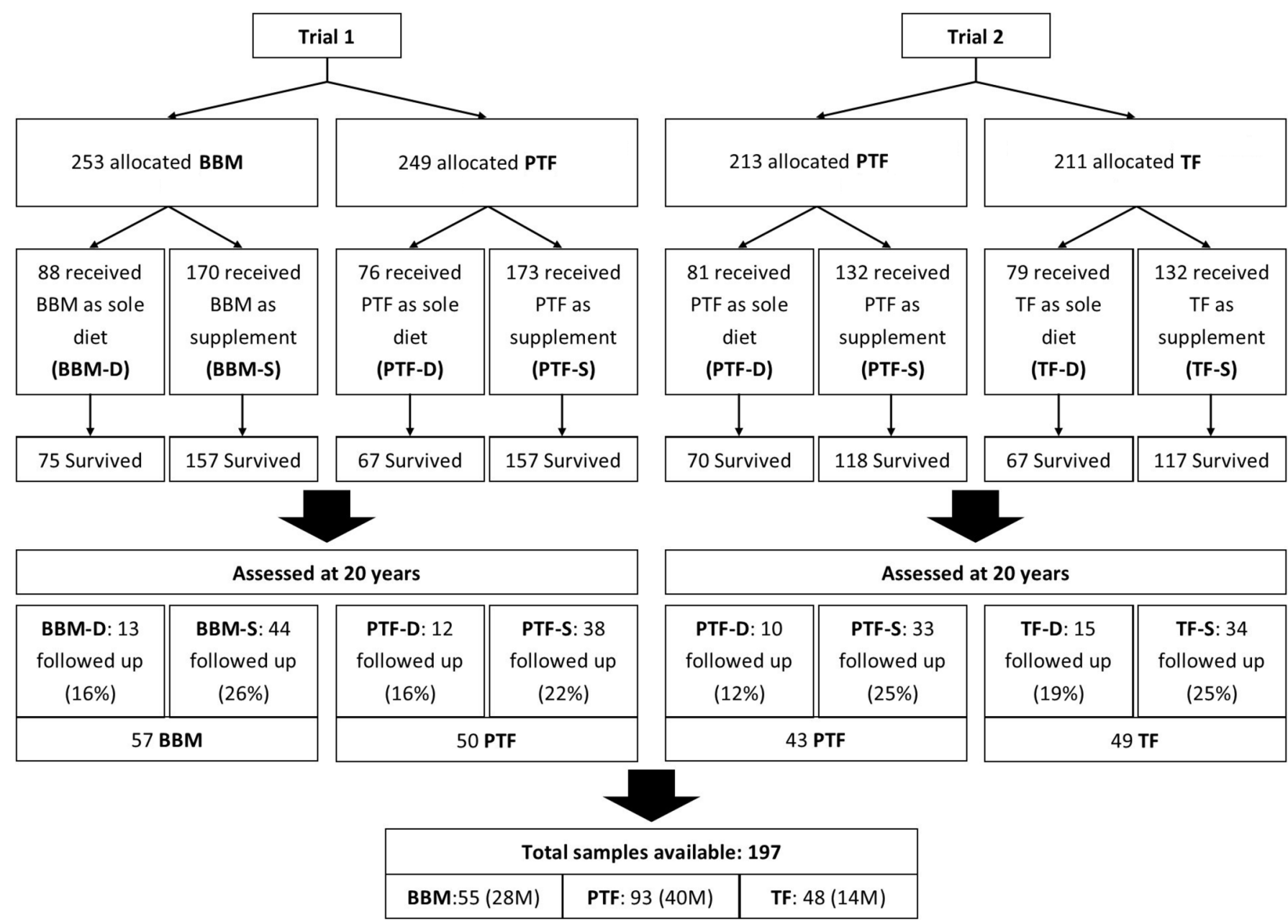

Figure 1 Original cohort details and recruitment data. Subjects were enrolled into one of two parallel trials and subsequently randomised to one of three different diets, preterm formula (PTF), term formula (TF), banked breast milk (BBM). Urine samples were collected during a follow-up study at age 20. 
Following parental consent, infants were randomised to one of three diets within 48 hours of birth: a) BBM, b) PTF (Osterprem, Farley's Health Products, Kendal, UK) or c) TF (Ostermilk, Farley's Health Products, Kendal, UK). Infants received this as their sole diet until their weight reached $2000 \mathrm{~g}$ or they were discharged home (median age 4 weeks). If a mother did not wish to provide her own breast milk (trial 1), infants (260 boys, 242 girls) were randomised to receive, as their sole diet, BBM or PTF (in Cambridge, Ipswich, King's Lynn: study 1); or to receive TF or PTF (in Norwich, Sheffield: study 2). If the mother wished to provide her own breast milk (trial 2), infants (203 boys, 221 girls) received as much maternal breast milk (MBM) as was available each day, and the rest of the diet was made up by randomising to the same options as trial 1.

Of the 926 infants enrolled in the neonatal trials, $831(89.7 \%)$ survived to discharge. Twenty years later, 272 young adults from this cohort were successfully contacted and invited to participate in a follow-up study investigating the effects of early diet on bone mass ${ }^{18}(70$ declined and the remainder could not be traced or did not reply); 202 participants were included in this study, representing $22 \%$ of those originally enrolled and $24 \%$ of known survivors. Urine samples were requested from all participants and obtained from a total of 197 (mean (SD) 20.25 (0.55) years). Urine samples were collected in standard NHS urine specimen pots without addition of antibacterial substances and stored immediately at $-80^{\circ} \mathrm{C}$.

Participants providing urine samples had been randomised for 1 month postnatally to BBM ( $\mathrm{n}=55$; 28 men), TF ( $\mathrm{n}=48 ; 14$ men) or PTF ( $\mathrm{n}=94 ; 40$ men). Participants in the follow-up study were more likely to be women and to have been classified in the highest social class category at birth. A greater proportion had been breast fed after discharge, although the median duration of any or exclusive breast feeding was not significantly different between follow-up participants and non-participants. There were no differences in indicators of neonatal disease severity or the proportion with a birth weight $<1250 \mathrm{~g}$ or below the 10th centile. Participants had a significantly reduced height SD score (SDS) and significantly higher body mass index $\operatorname{SDS}^{18}$ in comparison with the UK population reference values.

\section{High-resolution ${ }^{1} \mathrm{H}$-NMR spectroscopic analyses of urine samples}

Untargeted global metabolic profiles were acquired from 197 urine samples using ${ }^{1}$ H-NMR spectroscopy. Samples were prepared and standard one-dimensional spectral data acquired using a Bruker DRX-600 spectrometer (Bruker Biospin, Karlsruhe, Germany) operating at $600.29 \mathrm{MHz}$, according to published protocols. ${ }^{19}$ Samples were run at a temperature of $300 \mathrm{~K}$. Spectral preprocessing (phasing, baseline correction and reference to the TSP (trimethylsilyl-2,2,3,3-tetradeuteropropionic acid) singlet peak (at $\delta 0.00)$ ) were performed using the Bruker
TopSpin V.3.1 programme. Spectral data were imported into Matlab (V.R2014a, The MathWorks, Natick, Massachusetts, USA), and regions occupied by water and urea ( $\delta$ 4.45-6.00) removed. Data points were then aligned and normalised using the probabilistic quotient normalisation method ${ }^{20}$ to reduce the effects of differential dilution or concentration, on the data analysis (using scripts coded in-house in Matlab).

\section{Data analyses}

Spectroscopic data were analysed using both unsupervised and supervised multivariate statistical modelling methods (using proprietary Matlab scripts). Principal component analysis (PCA), an unsupervised approach, was conducted initially to discern the presence of inherent similarities or differences in urinary spectral profiles, and to identify outliers (based on positioning of points on the PCA scores scatter plot). The model's $\mathrm{R}^{2}$ statistic provides an indication of how much of the variation within a data set can be explained by the model components, with PC1 (principal component 1) explaining the greatest source of variation, followed by PC2, then PC3, and so on. The $\mathrm{Q}^{2}$ statistic is an indicator of a model's predictive ability, indicating how accurately the data, either classed or non-classed, can be predicted. The number of principal components used to build the models were based on optimal $\mathrm{R}^{2}$ and $\mathrm{Q}^{2}$ values (both of which vary between 0 and 1 ). PCA was followed by supervised modelling using orthogonal projections to latent structure discriminant analysis (O-PLS-DA), using the NMR spectroscopic data as $\mathrm{X}$ variables, and diet as the $\mathrm{Y}$ variable describing class ownership, to optimally model class differences corresponding to diet and to identify the metabolites contributing to this difference. ${ }^{21}$ Group comparisons were calculated for individuals on (1) high-calorie (PTF) versus low-calorie (TF and BBM combined) diets, (2) PTF vs BBM, (3) PTF vs TF for both the whole dataset and for men and women separately. In addition to these discrete models, we also conducted continuous modelling to identify metabolites correlated with birth weight, gestational age and 2-week weight $\mathrm{z}$ score. Multivariate modelling was conducted both on the whole dataset and on sex-stratified data.

\section{RESULTS}

\section{Principal component analysis}

The scores scatter plot from unsupervised PCA indicated that there was no strong biochemical signature in relation to the three neonatal nutritional regimens, as the points did not show any clustering or patterns relating to class (figure 2). Substantial variation in biochemical composition of participants' urine in this study was evidenced by the fact that the first two principal components only accounted for $34 \%$ of the total variation in the dataset (as would be expected in studies where no account has been made of dietary and lifestyle factors). Since PCA is a variance-based modelling method, these results indicate that 


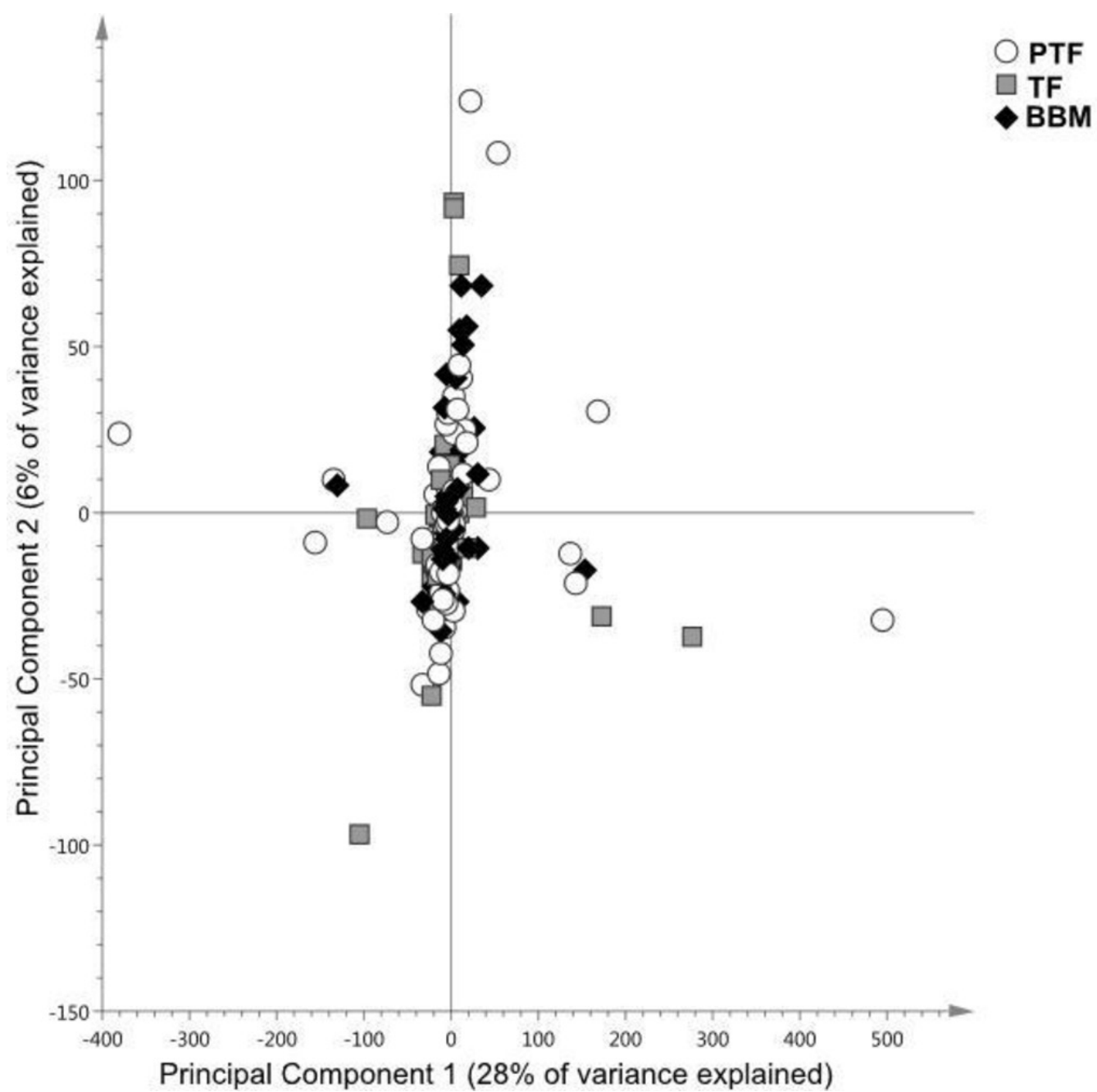

Figure 2 Principal components analysis (PCA) of urinary metabolic profiles in young adults born preterm. PCA scores scatter plot showing the distribution of urinary metabolic profiles in young adults born preterm, following different dietary regimes at birth (black diamonds=banked breast milk (BBM), grey squares=term formula (TF), white circles=preterm formula $(P T F)$ ) $(n=197)$. Number of principal components used to build the model $=2$ (cumulative $R^{2}=0.34$, cumulative $Q^{2}=0.31$ ).

multiple sources of variation were contributing to urinary composition, and that diet in the neonatal period alone was not a significant source of variation.

\section{Orthogonal projections to latent structure discriminant analysis}

In order to maximise the ability to detect any preterm diet-related differences in the data, O-PLS-DA of the urinary spectra data was used to make three dietary comparisons: 1) high-energy (PTF) versus low-energy diet (BBM and TF), 2) PTF versus BBM and 3) PTF versus TF. Models for sex-stratified data were also generated, in view of previous observations of sex-related differences in the predisposition of preterm infants to hypertension, fatty liver disease and metabolic syndrome. No significant models that could discriminate cleanly on the basis of nutritional intake were observed when spectra were analysed together or separately for men and women (table 1).

The $\mathrm{R}^{2} Y$ parameter indicated that the models accounted for between $66 \%$ and $94 \%$ of the total variance in the dataset, but the $\mathrm{Q}^{2} \mathrm{Y}$ values were negative, indicating that the models had no predictive power to differentiate individuals based on classification according to nutritional intake or birth weight and birth weight $\mathrm{z}$ score. One exception was the model associating gestational age with metabolic profiles, which delivered a model implying a weak association between metabolic profile and gestational age (explained variance $=65 \%$, predictive ability $26 \%$, table 1 ). This model was interrogated to identify the biomarkers responsible for the phenotypic relationship with gestational age and was found to be primarily influenced by ethanol excretion in 9 out of 197 individuals, which was biased towards the more mature gestational age group (see online supplementary figure $1)$. The ethanol resonances at $(\delta 1.18$ and $\delta 3.66)$ were removed and on remodelling the data, the $Q^{2} Y$ parameter indicated that the remodelled data fail to achieve predictive ability for any of the dietary group comparisons or correlation of metabolic data with clinical data, implying that we were unable to identify a distinct 
Table 1 O-PLS-DA analysis of urinary spectra in young adults born prematurely $R^{2} Y$ and $Q^{2} Y$ values for dietary group comparison and correlation of metabolic data with clinical data modelled on spectral data

\begin{tabular}{|c|c|c|c|c|c|c|}
\hline & \multicolumn{3}{|c|}{ Comparison of nutritional groups } & \multicolumn{3}{|c|}{ Correlation of metabolic data with clinical data } \\
\hline & $\begin{array}{l}\text { (1) } \\
\text { PTF vs (BBM+TF) }\end{array}$ & $\begin{array}{l}\text { (2) } \\
\text { PTF vs TF }\end{array}$ & $\begin{array}{l}\text { (3) } \\
\text { PTF vs BBM }\end{array}$ & Birth weight & $\begin{array}{l}\text { Birth weight } \\
\text { z score }\end{array}$ & Gestational age \\
\hline \multicolumn{7}{|c|}{ Combined } \\
\hline$R^{2} Y$ & 0.66 & 0.7 & 0.66 & 0.64 & 0.65 & 0.65 \\
\hline$Q^{2} Y$ & -0.6 & -0.66 & -0.38 & -0.38 & -0.27 & 0.26 \\
\hline \multicolumn{7}{|l|}{ Men } \\
\hline$R^{2} Y$ & 0.82 & 0.88 & 0.94 & 0.85 & 0.83 & 0.82 \\
\hline$Q^{2} Y$ & -0.63 & -0.54 & -0.57 & -0.11 & -0.5 & -0.24 \\
\hline \multicolumn{7}{|l|}{ Women } \\
\hline$R^{2} Y$ & 0.76 & 0.89 & 0.79 & 0.79 & 0.84 & 0.82 \\
\hline$Q^{2} Y$ & -0.4 & -0.38 & -0.33 & -0.4 & -0.14 & -0.32 \\
\hline
\end{tabular}

Data analysed combined and by gender $(n=197)$.

BBM, banked breast milk; O-PLS-DA, orthogonal projections to latent structure discriminant analyses; PTF, preterm formula; TF, term formula.

relationship between metabolic phenotype and gestational age (see online supplementary table 1 ).

\section{DISCUSSION}

We found no evidence of an effect of neonatal diet, allocated by randomisation, on the urinary metabolome of ex-preterm adults. The strength of our approach is that it represents the analysis of a non-invasively collected urine sample obtained at 20-year follow-up of participants in preterm neonatal randomised controlled trials in whom detailed information is available on functional outcomes. A weakness is that this analysis was not prespecified, and indeed could not have been, as the techniques employed were not available at the time the randomised controlled trials were conceived.

Clear differences in functional outcomes have been reported between the randomised groups, ${ }^{14} 1822$ hence it was plausible that differences might have been detected in the urinary metabolic profile. Several factors may have contributed to the lack of difference. Chief among these is variation introduced by recent diet, which has a strong influence on metabolic profile. ${ }^{23}$ Metabolism in humans is also heavily influenced by genetic and environment influences and their interactions, with phenotypes influenced by sex, age, stress, hormonal cycles, circadian rhythm and ethnicity. ${ }^{24}$ This extraneous variation is difficult to summarise, and hence adjust for, and can easily overshadow subtle effects that might relate to exposures that took place a long time ago. A weak correlation was initially observed in the model of gestational age and urinary profile but on examination of the correlation coefficients, the differences were driven by the higher excretion of ethanol and ethyl glucuronide by participants with a higher gestational age, indicating that alcohol consumption was higher in this group. This was particularly apparent for three individuals who biased the model. On exclusion of the ethanol-related resonances the model no longer positively correlated. Also of relevance to the lack of correlation with gestational age is that the range was narrow and included neither extremely immature $(<26$ weeks) or more mature ( $>34$ weeks) infants.

Our study was an opportunistic post hoc analysis that cannot exclude the possibility of differences in the preterm adult phenotype attributable to diet. The analysis was conducted using an NMR-based metabolic profiling strategy, and it is possible that other approaches (eg, using mass spectrometry hyphenated to chromatographic methods) may have yielded different results, particularly in identifying very subtle changes or signals that may have been overlapped in the NMR spectra, since these approaches offer the advantage of separation followed by detection. In previous work, we reported differences in the urinary metabolome between male term and ex-preterm adults based on a group size of $10-15 .^{25}$ This would imply that the present study might have been sufficiently powered to identify systematic effects of early diet if present. Furthermore, had the follow-up study collected blood from young adults in addition to urine, it is possible that there may have been additional findings since blood metabolic profiles typically reflect information on circulating metabolites, whereas urine predominantly provides information on intermediate and end point products of metabolism. We also acknowledge the possibility that the extended time span of 20 years with the introduction of multiple additional influences on phenotypic characteristics could only have served to further dilute power. Nonetheless, this study has demonstrated how high-throughput metabolic profiling offers considerable opportunity to detect metabolic signatures imprinted in infancy as shown by other groups. ${ }^{26}$ Our experience indicates that recommendations for future work include standardisation of urine collection (eg, in relation to time of day, menstrual cycle, 
preceding exercise and alcohol intake), and if possible diet, and consideration of power to detect prespecified and sex-specific differences.

Contributors JRCP, MJH, AS, AL and NM conceived the study. AS and AL ran the initial studies, and provided the samples for analysis. JRCP, AW, MJH and EH ran the sample analysis. NM and JP wrote the first draft of the paper. All authors contributed to subsequent drafts and and reviewed the final version.

Competing interests None declared.

Patient consent Obtained.

Ethics approval NHS Research Ethics Committee.

Provenance and peer review Not commissioned; externally peer reviewed.

Open Access This is an Open Access article distributed in accordance with the Creative Commons Attribution Non Commercial (CC BY-NC 4.0) license, which permits others to distribute, remix, adapt, build upon this work non-commercially, and license their derivative works on different terms, provided the original work is properly cited and the use is non-commercial. See: http://creativecommons.org/ licenses/by-nc/4.0/

(c) Article author(s) (or their employer(s) unless otherwise stated in the text of the article) 2017. All rights reserved. No commercial use is permitted unless otherwise expressly granted.

\section{REFERENCES}

1. Parkinson JR, Hyde MJ, Gale C, et al. Preterm birth and the metabolic syndrome in adult life: a systematic review and metaanalysis. Pediatrics 2013;131:e1240-63.

2. Singhal A, Kattenhorn M, Cole TJ, et al. Preterm birth, vascular function, and risk factors for atherosclerosis. Lancet 2001;358:1159-60.

3. Dietz WH. Critical periods in childhood for the development of obesity. Am J Clin Nutr 1994;59:955-9.

4. Lucas A. Programming by early nutrition in man. Ciba Found Symp 1991;156:38-50.

5. Crowther NJ, Cameron N, Trusler J, et al. Association between poor glucose tolerance and rapid post natal weight gain in seven-year-old children. Diabetologia 1998;41:1163-7.

6. Singhal A, Cole TJ, Fewtrell M, et al. Promotion of faster weight gain in infants born small for gestational age: is there an adverse effect on later blood pressure? Circulation 2007;115:213-20.

7. Ibáñez L, Lopez-Bermejo A, Suárez L, et al. Visceral adiposity without overweight in children born small for gestational age. J Clin Endocrinol Metab 2008;93:2079-83.

8. Forsén T, Eriksson J, Tuomilehto J, et al. The fetal and childhood growth of persons who develop type 2 diabetes. Ann Intern Med 2000;133:176-82.

9. Lucas A, Morley R, Cole TJ, et al. Early diet in preterm babies and developmental status at 18 months. Lancet 1990;335:1477-81.
10. Lucas A, Morley R, Cole TJ, et al. A randomised multicentre study of human milk versus formula and later development in preterm infants. Arch Dis Child Fetal Neonatal Ed 1994;70:F141-6.

11. Lucas A, Gore SM, Cole TJ, et al. Multicentre trial on feeding low birthweight infants: effects of diet on early growth. Arch Dis Child 1984;59:722-30.

12. Lucas A, Morley R, Cole TJ, et al. Early diet in preterm babies and developmental status in infancy. Arch Dis Child 1989;64:1570-8.

13. Lucas A, Morley R, Cole TJ. Randomised trial of early diet in preterm babies and later intelligence quotient. $B M J$ 1998;317:1481-7.

14. Singhal A, Cole TJ, Lucas A. Early nutrition in preterm infants and later blood pressure: two cohorts after randomised trials. Lancet 2001;357:413-9.

15. Singhal A, Fewtrell M, Cole TJ, et al. Low nutrient intake and early growth for later insulin resistance in adolescents born preterm. Lancet 2003;361:1089-97.

16. Singhal A, Faroogi IS, O'Rahilly $\mathrm{S}$, et al. Early nutrition and leptin concentrations in later life. Am J Clin Nutr 2002;75:993-9.

17. Nicholson JK, Lindon JC, Holmes E. 'Metabonomics': understanding the metabolic responses of living systems to pathophysiological stimuli via multivariate statistical analysis of biological NMR spectroscopic data. Xenobiotica 1999;29:1181-9.

18. Fewtrell MS, Williams JE, Singhal A, et al. Early diet and peak bone mass: 20 year follow-up of a randomized trial of early diet in infants born preterm. Bone 2009;45:142-9.

19. Beckonert O, Keun HC, Ebbels TM, et al. Metabolic profiling, metabolomic and metabonomic procedures for NMR spectroscopy of urine, plasma, serum and tissue extracts. Nat Protoc 2007;2:2692-703.

20. Dieterle F, Ross A, Schlotterbeck G, et al. Probabilistic quotient normalization as robust method to account for dilution of complex biological mixtures. Application in $1 \mathrm{H}$ NMR metabonomics. Anal Chem 2006;78:4281-90.

21. Cloarec O, Dumas ME, Trygg J, et al. Evaluation of the orthogonal projection on latent structure model limitations caused by chemical shift variability and improved visualization of biomarker changes in 1H NMR spectroscopic metabonomic studies. Anal Chem 2005;77:517-26.

22. Singhal A, Cole TJ, Fewtrell M, et al. Is slower early growth beneficial for long-term cardiovascular health? Circulation 2004;109:1108-13.

23. Esko T, Hirschhorn JN, Feldman HA, et al. Metabolomic profiles as reliable biomarkers of dietary composition. Am J Clin Nutr 2017; 105:547-54.

24. Bollard ME, Stanley EG, Lindon JC, et al. NMR-based metabonomic approaches for evaluating physiological influences on biofluid composition. NMR Biomed 2005;18:143-62.

25. Thomas EL, Parkinson JR, Hyde MJ, et al. Aberrant adiposity and ectopic lipid deposition characterize the adult phenotype of the preterm infant. Pediatr Res 2011;70:507-12.

26. Dessì A, Atzori L, Noto A, et al. Metabolomics in newborns with intrauterine growth retardation (IUGR): urine reveals markers of metabolic syndrome. J Matern Fetal Neonatal Med 2011;24(Suppl 2):35-9. 